Proceedings of the 2003 IEEE

International Conference on Robotics \& Automation

Taipei, Tajwan, September 14-19, 2003

\title{
Control of Biomimetic Locomotion via Averaging Theory
}

\author{
Patricio A. Vela ${ }^{\dagger \ddagger}$, Joel W. Burdick ${ }^{\ddagger}$ \\ ${ }^{\dagger}$ Control and Dynamical Systems $\quad \ddagger$ Mechanical Engineering \\ California Institute of Technology, Mail Code 107-81, Pasadena, CA, 91125 \\ pvela@cds.caltech.edu, jwb@robotics.caltech.edu
}

\begin{abstract}
Based on a recently developed "generalized averaging theory," we present a generic approach for the design of stabilizing feedback controllers for biomimetic locomotive systems. The control laws exponentialy stabilize in the average, and they apply to a very wide class of systems. Two examples are given: a "kinematic biped" that demonstrates how our theory handles discontinuities, and the snakeboard, which is an underactuated mechanical system with drift.
\end{abstract}

\section{Introduction}

Most mobile robots use wheels, as they provide the simplest means for mobility. However, wheels are undesirable for a number of potential applications. In these situations, we can borrow ideas from nature. Biomimetic locomotion refers to the movement of robotic mechanisms in ways that are analogous to the movement patterns of natural organisms. Biomimetic propulsion is typically generated by a coupling of periodic body deformations to external constraints. The forces generated by these constraint interactions induce net robot movement.

This paper presents a generic feedback control strategy for biomimetic robotic locomotors. Our approach is based on a recently developed generalized averaging theory that analyzes, to arbitrary order, the dynamics of systems undergoing periodic motion [17]. Using this averaging theory, we have developed general feedback control strategies for underactuated dynamical systems [16]. This paper shows how to apply these theories to biomimetic locomotors, which are generically underactuated dynamical systems driven by periodic inputs.

To illustrate these ideas, our theory is applied to two examples: a "kinematic biped" and the "Snakeboard". These cxamples illustrate the utility of our approach in a variety of contexts. The biped uses piecewise holonomic constraints to achieve motion, whereas the snakeboard uses nonholonomic constraints. The snakeboard's dynamics are smooth, while the biped's are discontinuous. Our biped model is inspired by the work of Ito et. al. [14], who examine an underactuated crawling robot. They did perform a controllability analysis, but did not analyze their feedback strategy. Bullo and Lewis [9] have developed a motion control strategy for the snakeboard based on kinematic motions, i.e., those that start and

\footnotetext{
${ }^{1}$ This work was supported in part by the National Science Foundation by Engineering Research Center grant NSF9402726.
}

end at zero velocity. When applied to this model, our control approach is not limited to kinematic motions, and additionally enables trajectory tracking.

There is a vast literature on the analysis and control of biomimetic robotic locomotion. Prior efforts have focused on a particular morphology (e.g. biped [12], quadruped [6], or carangiform fish), or on particular locomotion modes (e.g. quasi-static [21] or hopping [20]). In contrast, our approach works across a large range of morphologies and locomotion modes. This work springs from a line of work aimed at developing a comprehensive framework for biomimetic locomotion engineering. For example, Ref. [13] showed how the mechanics of a large class of undulatory locomotors can be treated in a systematic way. Ref.s $[4,5]$ showed how to use stratified configuration space to extend these ideas to the analysis and open loop control of legged locomotion. What has been missing in this line of work is a general strategy for feedback control. This paper fills that gap. We note that an earlier version of this theory here has been experimentally verified on a robotic fish [15].

The closest prior work in the field of robotics uses the Poincaré map to analyze the dynamics and control strategies for simple legged robot models $[7,23]$ undergoing periodic motion. In [22], amplitude modulation was used to obtain a stable Poincaré map for an underactuated planar manipulator. This prior work was practically limited to very simple dynamical models, and had no systematic procedure for control design. The method reviewed here overcomes these limitations.

Our control approach extends and generalizes the vast literaturc on control of nonholonomic and underactuated mechanical systems. For a review of relevant prior work in these fields, see the literature review in $[17,16]$.

Section 2 reviews the averaging theory developed in [17], summarizes the transformations required to place periodically forced $2^{\text {nd }}$-order systems into the averaging form, and reviews the control design approach [16]. Section 3 , introduces the kinematic biped model, and applies our theory to demonstrate feedback control. Section 4 discusses feedback control of the snakeboard. 


\section{A General Averaging Theory}

Generically, the governing mechanics of many biomimetic locomotors may take the form:

$$
\dot{x}=X(x, t ; \epsilon)=\epsilon \widehat{X}(x, t), \quad x(0)=x_{0},
$$

with $X$ being $T$-periodic, i.e., $X(x, t ; \epsilon)=X(x, t+T ; \epsilon)$, and where $X(\cdot, t)$ may be written as $X_{t}$.

Theorem 1 (Nonlinear Floquet Theorem) [17] Let $\Phi_{0, t}^{X}$ be the flow of the time-periodic differential equation (1). If the monodramy map has a logarithm, then the flow $\Phi_{0, t}^{X}$ can be represented as a composition of flows $\Phi_{0, t}^{X}=P(t) \circ \exp (Y t) \mathrm{Id}$, where $P$ is $T$-periodic.

The monodramy map is the flow of $X$ at time $T$, e.g., $\Phi_{0, T}^{X}$. It coincides with the flow of the autonomous vector field, $Y$, at time $T$, e.g., $\exp (Y T)$. This theorem says that the flow of $(1)$ can be decomposed into the composition of two flows: (1) the flow of an autonomous vector field; $Y$, that represents the average system behavior; and (2) a periodic oscillation about this flow.

Theorem 2 [17] If the monodramy map has a fixed point, the actual flow has a periodic orbit whose stability is determined by the stability of the monodramy map.

Corollary 1 [17] If the flow of system (1) has a fixed point $x^{*}$, as does the monodramy map, stability of the actual flow may be determined the using monodramy map. A linearly (asymptotically) stable fixed point for the monodramy map implies a linearly (asymptotically) stable fixed point for the actual system.

Thus, for the cases of interest to this paper, we can infer the stability of the original system (1) from the stability of the monodramy map. Our averaging theory finds suitable approximations to this flow from truncations of the flow's infinite series expansion.

The series expansions described in [1] seek to make sense of the flow of system (1), and do so in a manner consistent with the nonlinear Floquet theorem. The flow can be written as an infinite series given by the chronological exponential,

$$
\Phi_{0, t}^{X}=\overrightarrow{\exp }\left(\int_{0}^{t} X_{\tau} \mathrm{d} \tau\right)
$$

A decomposition is sought such that there exists an autonomous vector field, $\overrightarrow{\mathrm{V}}_{0, t}\left(X_{\tau}\right)$, whose flow after unit time equals the flow of the original system at time $t$; more precisely,

$$
\overrightarrow{\exp }\left(\int_{0}^{t} X_{\tau} \mathrm{d} \tau\right)=\exp \left(\overrightarrow{\mathrm{V}}_{0, t}\left(X_{\tau}\right)\right)
$$

Changes in the final time yield a new autonomous flow. The solution to this equation is given by the logarithm,

$$
\vec{V}_{0, t}\left(X_{\tau}\right)=\ln \overrightarrow{\exp }\left(\int_{0}^{t} X_{\tau} \mathrm{d} \tau\right),
$$

and is an infinite series. For the case of a time-periodic vector field, the autonomous vector field evaluated at final time $t=T$ is related to the average vector field of $X_{t}$.

$$
Y=\frac{1}{T} \ln \overrightarrow{\exp }\left(\int_{0}^{T} X_{\tau} \mathrm{d} \tau\right)=\frac{1}{T} \overrightarrow{\mathrm{V}}_{0, T}\left(X_{\tau}\right)
$$

Definition 1 If the function $\mathrm{F}$ can be given by a series expansion, then Trunc ${ }_{m}(\mathrm{~F})$ is a truncation of the series of the $(m+1)$ and higher terms.

The theorems below relate the truncated versions to the full expansions.

Theorem 3 [17] The $\mathrm{m}^{\text {th }}$-order truncation of the logarithm of the monodramy map gives an $(\mathrm{m}+1)^{\text {th }}$-order approximation of the flow for finite time, i.e.,

$$
\exp (Y t)=\exp \left(Y^{m} t\right)+O\left(\epsilon^{m+1}\right)
$$

on the time scale 1 .

Theorem 4 [17] An $\mathrm{m}^{\text {th }}$-order truncation of the timeperiodic Floquet mapping is of order $(m+1)$-close to the time-periodic Floquet mapping on the time scale 1.

$$
P(t)=\operatorname{Trunc}_{m}(P(t))+O\left(\epsilon^{m+1}\right)
$$

Proposition 1 [17] If the Floquet mapping has a timeindependent bias, i.e., $P(t)=\widetilde{P}(t) \circ P_{0}$. Then the new averaged vector field may be written $Z=\left(P_{0}\right)_{*} Y$.

The evolution of the Floquet solution becomes, $x(t)=$ $\widetilde{P}(t) \circ \exp (Z t) x_{0}$.

\subsection{Second Order Systems}

The equations of motion governing second order mechanical systems (e.g. the snakeboard) take the form:

$$
\ddot{q}=\widetilde{X}(q, \dot{q}, t) .
$$

Converting to $1^{\text {st }}$-order state form, $x=(q, \dot{q})$, and assuming that the controls consist of a state feedback, $u^{a}(x)$, and periodic, $v^{a}(t)$, components, we obtain:

$$
\dot{x}=S(x)+X_{a}(x) u^{a}(x)+\frac{1}{\epsilon} X_{a}(x) v^{a}\left(\frac{t}{\epsilon}\right) .
$$

To place this into the averaging form, transform time,

$$
\frac{\mathrm{d} x}{\mathrm{~d} \tau}=\epsilon\left(S(x)+X_{a}(x) u^{a}(x)\right)+X_{a}(x) v^{a}(\tau),
$$

then utilize the variation of constants formula,

$$
x(t)=\Phi_{0, t}^{X_{a}(x) v^{a}(\tau)} \circ \Phi_{0, t}^{Z}
$$


where,

$$
Z=\epsilon\left(\Phi_{0, \tau}^{X_{a}(x) v^{a}(\tau)}\right)^{*}\left(S(x)+X_{a}(x) u^{a}(x)\right)
$$

where $(\cdot)^{*}$ denotes pull-back. The flow $\Phi_{0, \tau}^{X_{a}(x) v^{\alpha}(\tau)}$ was shown to be periodic in [8], implying that the vector field $Z$ is also periodic. The vector field $Z$ is also in the form required by averaging theory. Applying the Floquet theorem, and transforming time,

$$
x(t)=\Phi_{0, t / \epsilon}^{X_{a}(x) v^{a}(\tau)} \circ P(t / \epsilon) \circ \exp (-Y t / \epsilon)
$$

where $Y$ is the autonomous averaged vector field corresponding to $Z$. Thus, the evolution of $x(t)$ obeys,

$$
x(t)=P_{2}(t / \epsilon) \circ \exp (-Y t / \epsilon)
$$

where $P_{2}(t)=\Phi_{0, t}^{X_{a}(x) v^{a}(\tau)} \circ P(t)$ and is periodic since its constitutive flows are periodic. Thus, second order systems under high-amplitude, high-frequency forcing can be placed within the paradigm of averaging theory.

\subsection{Averaging for Mechanical Systems}

We now apply this theory to derive canonical averaged forms for mechanical systems undergoing periodic forcing. First and second order averages are derived from appropriate truncations of the vector field expansions. An underactuated driftless affine control system with timeperiodic actuation will take the form:

$$
\dot{q}=Y_{a}(q) v^{a}(t / \epsilon),
$$

with periodic control inputs, $v(t)=v(t+T)$. The $1^{s t}$ order average is:

$$
\dot{q}=Y_{a}(q) \overline{v^{a}(t)},
$$

where $\overline{v(t)}=\frac{1}{T} \int_{0}^{T} v(t) \mathrm{d} t$. The second order average is:

$$
\dot{q}=Y_{a}(q) \overline{v^{a}(t)}+\frac{1}{2} \epsilon \overline{\mathrm{V}_{(1,0)}^{(a, b)}(t)}\left[Y_{a}(q), Y_{b}(q)\right],
$$

where $[\cdot, \cdot]$ denotes the Lie bracket of vector fields, and the averaged coefficient, $\overline{V_{(1,0)}^{(a, b)}(t)}$, is

$$
\overline{V_{(1,0)}^{(a, b)}(t)}=\frac{1}{T} \int_{0}^{T} \int_{0}^{t} v^{a}(\tau) v^{b}(t) \mathrm{d} \tau \mathrm{d} t
$$

For systems with drift, Equation (8) has a different $1^{\text {st }}$. order averaged structure ${ }^{1}$

$$
\dot{x}=S(x)+Y_{0}(x)-\frac{1}{2} \overline{\mathrm{V}_{(i, 1)}^{(a, b)}(t)}\left\langle Y_{a}: Y_{b}\right\rangle,
$$

where the averaged coefficient, $\overline{V_{(1,1)}^{(a, b)}(t)}$, is

$$
\overline{\mathrm{V}_{(1,1)}^{(a, b)}(t)}=\frac{1}{T} \int_{0}^{T} \int_{0}^{t} v^{a}(\tau) \mathrm{d} \tau \int_{0}^{t} v^{b}(\tau) \mathrm{d} \tau \mathrm{d} t .
$$

\footnotetext{
${ }^{1}$ We do not need higher order averages for the purposes of this paper. See $[16]$ for their structure.
}

The $S(x)$ term contains the natural unforced dynamics of the system, while the $Y_{0}$ term contains the stablizing control strategy for the directly controlled states. The symmetric product, $\left\langle Y_{a}: Y_{b}\right\rangle$ is a result of the variation of constants formula. It is defined as,

$$
\left\langle Y_{a}: Y_{b}\right\rangle \equiv\left[Y_{a},\left[S+Y_{0}, Y_{b}\right]\right]
$$

For details of the averaged equations for systems without and with drift, see [16] and [18], respectively.

\subsection{Stabilization via Averaging}

The averaged equations consist of the terms corresponding to the directly controllable vectors fields and Lie brackets. If the system demonstrates controllability via the use of these brackets, then the choice of suitable periodic forcing can cause the system to flow in arbitrary directions. The construction of these inputs is beyond the scope of the article, and more information can be found in $[16,18]$. The construction process will lead to a set of undetermined coefficients $\alpha$ that scale the periodic inputs. The parameters $\alpha$ modulate the degree of flow in the direction of the Lie brackets that they multiply.

For driftless systems under time-periodic actuation, the general averaging theory leads to

$$
\dot{z}=B(z) H(\alpha)
$$

where the matrices $B$ and $H$

$$
B(z)=\left[\widehat{\mathrm{Y}}_{1} \ldots \widehat{\mathrm{Y}}_{N}\right] \text { and } H(\alpha)=\left[\mathrm{T}^{1} \ldots \mathrm{T}^{N}\right] \text {, }
$$

consist of the input vector fields and their Lie brackets, and the corresponding averaged coefficients, respectivcly.

Theorem 5 [19] Consider a driftless system (14) which is small time locally controllable. Let $u^{k}(t)$ be the set of $\alpha$ parametrized, $T$-periodic input functions where $k=$ $\{1, \ldots, m\}$ and $\alpha \in \mathbb{R}^{n-m}$. Let $z(t)$, be the averaged system response to the inputs. Given the averaged system (19), assuming that the $m$ directly controlled states have been linearly stabilized and that the linearization of $H$ with respect to $\alpha$ at $\alpha=0$ and $z=z^{*}$ is invertible on the $(n-m)$ dimensional subspace to control, there exists a $K \in \mathbb{R}^{(n-m) \times n}$ such that for

$$
\alpha=-\Lambda K z(T\lfloor t / T\rfloor)
$$

where $\Lambda^{(n-m) \times(n-m)}$ is invertible and $\lfloor\cdot\rfloor$ denotes the floor function, the average system response is stabilized.

The proof of this theorem is based on the linearization of the system, and integration of the linearized system over one period to obtain a discretized model. Appropriate feedback gains stabilize the discrete system. The theorem finds gains such that the monodramy map is linearly stable, so the original system is also linearly stabilized. Stabilization to an orbit or to a fixed point will depend on the nature of the input set, and whether it satisfies only Theorem 2 or if it also satisfies Corollary 1 . 
For second order systems with drift, the general averaging theory lcads to equations with the structure:

$$
\dot{z}=S(z)+Y_{0}(z)+B(z) H(\alpha)
$$

where the matrices $B$ and $H$,

$$
B(z)=\left[\widehat{\mathrm{Y}}_{1} \ldots \widehat{\mathrm{Y}}_{N}\right] \text { and } H(\alpha)=\left[\mathrm{T}^{1} \ldots \mathrm{T}^{N}\right],
$$

consist of the input vector fields and their symmetric products, and the corresponding averaged coefficients, respectively. A theorem exactly analogous to Theorem 5 holds for the second order case [18].

\section{Kinematic Biped}

As a demonstration of the theory, we consider a "kinematic biped" that is motivated by the underactuated crawling robot of Ito et. al. [14]. The model (see Fig. 1) operates on flat ground. When either of the two vertically moving peg-legs contact the ground, we assume the crawler pivots about the contact. Casters on the four corners provide balance, thereby planarizing the motion. A rotating momentum wheel is the main motion generator. It is hereafter called the rotor. By properly switching the feet contacts in phase with the rotor's spin, the biped can maneuver.


Figure 1: Top and Side Views of the Kinematic Biped

In kecping with the language of [13], the configuration space, $Q$, is the principal bundle $Q=G \times B$, where $G$ and $B$ denote the group and shape spaces. The shape variables are the rotor angle, $\psi$, and the feet state, $N=$ $\{L, R\}$. The symbols $L$ and $R$ denote when the left or right foot is put down. The group is $G=S E(2)$, the set of planar rigid body motions.

The equations of motion are derived using the approach of Ref. [3]. This method uses the principal bundle c-space structure and the group invariance of the Lagrangian and constraints to compute the "reduced" Euler-Lagrange equations. For those not familiar with this approach, we appeal to the physicial intuition behind the mathematical concepts during the derivation.

The Lagrangian for the system is:

$$
L(q, \dot{q})=\frac{1}{2} M\left(\dot{x}^{2}+\dot{y}^{2}\right)+\frac{1}{2} J \dot{\theta}^{2}+\frac{1}{2} J_{m}(\dot{\theta}+\dot{\psi})^{2},
$$

where $M$ is the robot's total mass, $J\left(J_{m}\right)$ is the inertia of the body (rotor). When the right leg is planted, the resulting holonomic constraint on the robot's motion is:

$$
\dot{x}=d \sin \theta \dot{\theta}, \text { and } \dot{y}=-d \cos \theta \dot{\theta} .
$$

Since the Lagrangian and constraints are $G$-invariant, they may be reduced to the Lie algebra; equivalent to giving a description in body coordinates. The reduced constraints are,

$$
\xi^{1}=0 \text { and } \xi^{2}=-d \xi^{3} .
$$

Since the constraints span 2 dimensions of the 3 dimensional Lie algebra, $\mathfrak{s e}(2)$, Noether's theorem implies a conserved momentum (about the foot's axis). Conservation of momentum is given more abstractly by the equation, $\mathcal{A}^{n h c} \equiv\left(\mathbb{I}^{c}\right)^{-1} \mathrm{~J}^{n h c}=\left(\mathbb{I}^{c}\right)^{-1} p$, where $\mathbb{I}^{c}$ is the inertia of the system and $p$ is the conserved momentum. More explicitly,

$$
M d \xi^{2}+\left(J+J_{m}\right) \xi^{3}+J_{m} \dot{\psi}=p .
$$

This equation is combined with the kinematic constraints to obtain the constraint equations,

$$
\mathrm{E}(r) \xi+\mathrm{F}(r) \dot{r}=\mathrm{G}(r) p
$$

where,

$$
\mathrm{E}(r)=\left[\begin{array}{ccc}
1 & 0 & 0 \\
0 & 1 & d \\
0 & M d & J+J_{m}
\end{array}\right] \mathrm{F}(r)=\left[\begin{array}{c}
0 \\
0 \\
J_{m}
\end{array}\right] \quad \mathrm{G}(r)=\left[\begin{array}{l}
0 \\
0 \\
1
\end{array}\right]
$$

The local connection form, describing the system constraints in body coordinates, is found by solving for the Lie algebra element, $\xi$, giving the reconstruction equation,

$$
\xi=-\mathcal{A}_{l o c}(r) \dot{r}+\Gamma_{l o c}(r) p,
$$

with the local connection and the affine forms equal to,

$$
\begin{aligned}
& \mathcal{A}_{\text {loc }}(r)=\frac{J_{m}}{J+J_{m}+M d^{2}}\left[\begin{array}{c}
0 \\
-d \\
1
\end{array}\right], \text { and } \\
& \Gamma_{l o c}(r)=\frac{1}{J+J_{m}+M d^{2}}\left[\begin{array}{c}
0 \\
-d \\
1
\end{array}\right] .
\end{aligned}
$$

Continuing with the method of [3], one defines an orthogonal basis (in the Lagrangian metric) for the Lie algebra:

$$
e_{1}(r)=\left[\begin{array}{c}
0 \\
-d \\
1
\end{array}\right], \quad e_{2}(r)=\left[\begin{array}{l}
1 \\
0 \\
0
\end{array}\right], \quad e_{3}(r)=\left[\begin{array}{c}
0 \\
\frac{J+J_{m}}{M d} \\
1
\end{array}\right] .
$$

The basis is used to derive the momentum equation,

$$
\frac{\mathrm{d}}{\mathrm{d} t} p_{1}=0,
$$

and also the shape space dynamics,

$$
\left(\frac{1}{2} J_{m}+\frac{3}{2} \frac{J_{r}^{2}}{J_{b}+J_{r}+M d^{2}}\right) \ddot{\psi}=r,
$$

where $\tau$ is the torque on the rotor. For zero initial momentum, the reconstruction equation becomes

$$
\xi=-\mathcal{A}_{\text {loc }}(\psi) \dot{\psi}
$$


where $\mathcal{A}_{l o c}(\psi)$ will be one of two choices, according to which foot ${ }^{2}$ is on the ground

$$
\begin{gathered}
\text { right : } \mathcal{A}_{l o c}(\psi)=\frac{J_{m}}{J+J_{m}+M d^{2}}\left[\begin{array}{c}
0 \\
d \\
-1
\end{array}\right], \\
\text { left : } \mathcal{A}_{l o c}(\psi)=\frac{-J_{m}}{J+J_{m}+M d^{2}}\left[\begin{array}{l}
0 \\
d \\
1
\end{array}\right] .
\end{gathered}
$$

Locomotion and Controllability By appropriate cycling of the feet and phasing of the rotor, it is possible to (i) rotate in place (ii) move forward (iii) move laterally. The existence of these motions can be shown by our averaging theory. Choosing $\dot{\psi}$ and the foot placements as inputs, Equation (25) takes the form

$$
\xi=\mathbb{A}_{1} v^{1}(t)+\mathbb{A}_{2} v^{2}(t)
$$

where

$$
\mathbb{A}_{1}=\left(\mathcal{A}_{\text {loc }}\right)_{\text {right }} \quad, \quad \mathbb{A}_{2}=\left(\mathcal{A}_{\text {loc }}\right)_{\text {left }},
$$

and the functions $v^{1}(t)$ and $v^{2}(t)$ cannot be non-zero simultaneously. Further, we assume that foot placement transitions occur when at the robot is at rest. Since the functions $v^{a}(t)$ will be chosen to be periodic functions of time, we can use averaging theory to analyze the crawler's motion.

To demonstrate the crawler's controllability, note that the two distinct foot placement possibilities represent two linearly independent vectors in the Lie algebra. A third linearly independent possibility must exists for controllability. From Bullo and Zefran [10], it suffices to show that the Lie bracket between the two motions results in a lineary independent direction. Since

$$
\left[\mathbb{A}_{1}, \mathbb{A}_{2}\right]=-\left[\begin{array}{lll}
2 d & 0 & 0
\end{array}\right]^{T}
$$

the system is configuration controllable, therefore enabling point-to-point repositioning and reorientation. Matsuno et. al. [11] reached the same conclusion for a similar crawling robot, but without utilizing the inherent symmetries of the system to reduce to the Lie algebra. The geometry of this biped also avoids the singularity associated with their robot analysis.

Forward Gait For forward motion, let $\dot{\psi}(t)=\sin (t)$. The right foot starts in the down position, while the left foot is placed at $t=\pi$, when the rotor reverses direction. The periodic functions $v^{\alpha}(t)$ decompose accordingly,

$$
\begin{aligned}
& v^{1}(t)= \begin{cases}\sin (t) & \text { for } 0 \leq t<\pi \\
0 & \text { for } \pi \leq t \leq 2 \pi\end{cases} \\
& v^{2}(t)= \begin{cases}0 & \text { for } 0 \leq t<\pi \\
\sin (t) & \text { for } \pi \leq t \leq 2 \pi\end{cases}
\end{aligned}
$$

\footnotetext{
${ }^{2}$ The calculations for the opposite foot merely changes a sign on the constraints. The trivial dynamics of the momentum state are not modified, nor are the shape space dynamics.
}

A non-zero average, implies that first order averaging theory will suffice. The average is,

$$
\eta=\mathbb{A}_{1} \overline{v^{1}(t)}+\mathbb{A}_{1} \overline{v^{2}(t)}=\mathbb{A}_{1} \frac{1}{\pi}-\mathbb{A}_{2} \frac{1}{\pi}=\left[\begin{array}{c}
0 \\
\frac{2 d}{\pi} \\
0
\end{array}\right]
$$

Rotate Gait Rotational motion is similar, but does not require a reversal of the rotor's spin direction, i.e., $\dot{\psi}=1-\cos (2 t)$. The input functions are

$$
\begin{aligned}
& v^{1}(t)= \begin{cases}1-\cos (2 t) & \text { for } 0 \leq t<\pi \\
0 & \text { for } \pi \leq t \leq 2 \pi\end{cases} \\
& v^{2}(t)= \begin{cases}0 & \text { for } 0 \leq t<\pi \\
1-\cos (2 t) & \text { for } \pi \leq t \leq 2 \pi\end{cases}
\end{aligned}
$$

The first order average is,

$$
\eta=\mathbb{A}_{1} \overline{v^{1}(t)}+\mathbb{A}_{1} \overline{v^{2}(t)}=\mathbb{A}_{1} \frac{1}{2}+\mathbb{A}_{2} \frac{1}{2}=\left[\begin{array}{l}
0 \\
0 \\
1
\end{array}\right]
$$

Sideways Gait This gait is subtler, as it arises from a higher order dynamical effect. The rotor follows the function

$$
\dot{\psi}(t)=\left\{\begin{aligned}
\sin (2 t) & 0 \leq t<\pi \\
-\sin (2 t) & \pi \leq t \leq 2 \pi
\end{aligned}\right.
$$

with foot placements occuring at the zero crossings of this function. The corresponding input functions are,

$$
\begin{aligned}
& v^{1}(t)=\left\{\begin{aligned}
\sin (2 t) & 0 \leq t \leq \pi / 2 \\
-\sin (2 t) & \pi \leq t \leq 3 \pi / 2 \\
0 & \text { otherwise }
\end{aligned}\right. \\
& v^{2}(t)=\left\{\begin{aligned}
\sin (2 t) & \pi / 2 \leq t \leq \pi \\
-\sin (2 t) & 3 \pi \leq t \leq 2 \pi \\
0 & \text { otherwise }
\end{aligned}\right.
\end{aligned}
$$

The averages of these functions vanish. Hence, $2^{\text {nd }}$-order averaging methods must be used [16]. The averaged system evolution is given by

$$
\xi=\frac{1}{2} \overline{V_{(1,0)}^{(a, b)}(t)}\left[\mathbb{A}_{a}, \mathbb{A}_{b}\right]
$$

where the only non-vanishing second order averaged coefficent, $\overline{\mathrm{V}_{(1,0)}^{(a, b)}(t)}$, is:

$$
\overline{\mathrm{V}_{(1,0)}^{(1,2)}(t)}=\frac{1}{T} \int_{0}^{T} \int_{0}^{t} v^{1}(\tau) \mathrm{d} \tau v^{2}(t) \mathrm{d} t=-\frac{1}{2 \pi},
$$

and it's skew-symmetric partner, $\overline{V_{(1,0)}^{(2,1)}(t)}$. The averaged evolution of the system is,

$$
\begin{aligned}
\eta=\frac{1}{2} \overline{V_{(1,0)}^{(1,2)}}\left[\mathbb{A}_{1}, \mathbb{A}_{2}\right]+\frac{1}{2} \overline{V_{(1,0)}^{(2,1)}}\left[\mathbb{A}_{2}, \mathbb{A}_{1}\right] \\
=-\frac{1}{4 \pi} \cdot\left[\begin{array}{c}
-2 d \\
0 \\
0
\end{array}\right]+\frac{1}{4 \pi} \cdot\left[\begin{array}{c}
2 d \\
0 \\
0
\end{array}\right]=\left[\begin{array}{c}
\frac{d}{\pi} \\
0 \\
0
\end{array}\right],
\end{aligned}
$$

which is sideways translation for the discrete crawler.

The various gaits are simulated in Fig 2. Feedback control will be added below to correct for existing gait errors and other disturbances. 


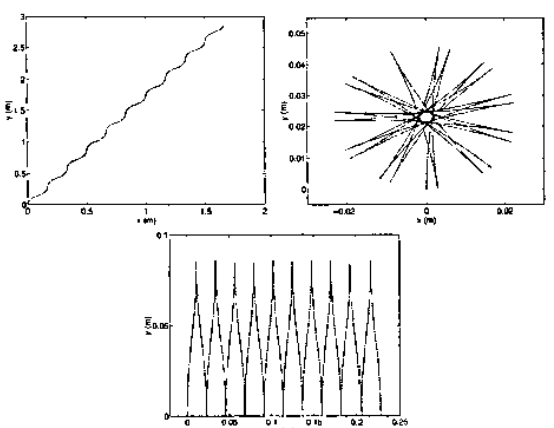

Figure 2: Gaits for the Discrete Crawler

Stabilization Define the rotor's control function to be

$$
\psi(t)=\alpha_{1} \sin (t)+\frac{1}{2} \alpha_{2}(1-\cos (2 t))
$$

with $\alpha_{1}$ and $\alpha_{2}$ to be chosen. The foot constraints transition at $t=(2 k+1) \pi, k \in \mathbb{Z}$. The parameter $\alpha_{1}$ controls forward motion, while $\alpha_{2}$ controls rotational motion, allowing a superposition of the forward and rotate gaits.

The configuration error is defined to be $g_{\text {err }} \equiv g^{-1} g_{d e s}$, where $g_{d e s} \in S E(2)$ is the goal configuration and $g \in$ $S E(2)$ the current configuration. Define error functions

$$
\begin{aligned}
& \alpha_{1}=k_{2} y_{e r r} \\
& \alpha_{2}=k_{1} \tan ^{-1}\left(x_{e r r} / y_{e r r}\right)+k_{3} \theta_{e r r} .
\end{aligned}
$$

where the $k_{i}$ are chosen according to Theorem 5 . Note that when the configuration error is zero, the terms $\left(\alpha_{1}, \alpha_{2}\right)$ vanish. Hence, both the original and averaged systems share the same equilibria. Therefore, via Corollary 1 , the system exponentially stabilizes to the goal, achieving point-to-point repositioning.

Using the gains $k=(3,8,0)$ results in the stabilizating trajectory pictured in Fig. 3 . The biped started at $(x, y, \theta)=\left(-1,5, \frac{\pi}{5}\right)$ and was commanded to the origin. Fig. 4 shows superimposed snapshots from the simulation. The small dot on either side of the biped's body represents the active foot constraint. The gridlines are spaced 1 unit apart. Orientation control can also be included, but would require a slightly more sophisticated controller.

Our stabilization theorem also allows for trajectory tracking - the goal point is time varying. Fig. 5 demonstrates trajectory tracking for a straight path. In Fig. 6 the crawler must track the same trajectory, but starts with a large initial error of $(x, y, \theta)=\left(-1,5, \frac{\pi}{5}\right)$. It successfully moves toward the trajectory, then proceeds to track it. There is a small steady state error.

\section{The Snakeboard}

Since the Snakeboard model was introduced and described in detail in [2], we only summarize here its equations. The snakeboard represents an underactuated system with drift--traditionally a difficult control problem.
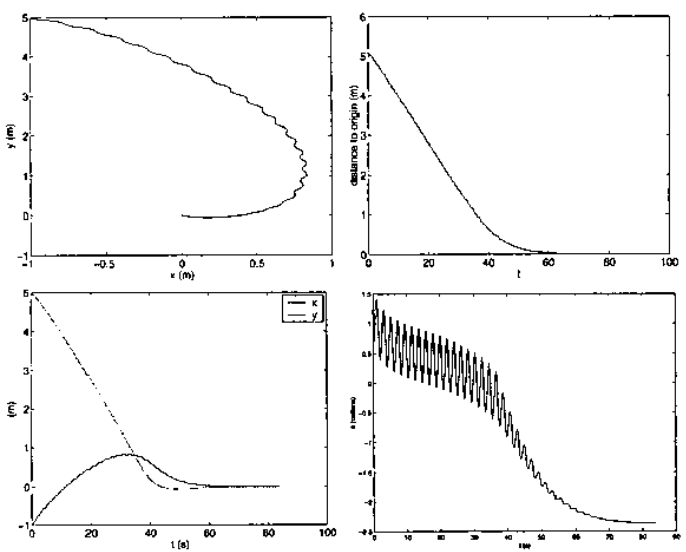

Figure 3: Point Stabilization; Parametric plot, Magnitude plot, $x(t)$ and $y(t)$, and $\theta(t)$ Plots

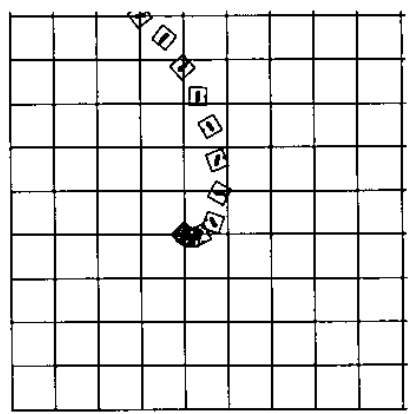

Figure 4: Snapshots of Point Stabilization

In the simplified model used here, the snakeboard consists of a rigid body to which there is attached a momentum wheel (or rotor), and two pairs of wheels. The wheels axles are coupled to the main body by independently controllable revolute joints (see Fig. 7). (Fig. 7). The c-space for this system is $\left[x, y, \theta, \psi, \phi_{b}, \phi_{f}\right] \in$ $S E(2) \times \mathbb{T}^{3}$ where $g=[x, y, \theta]^{T}$ is the position and orientation of the board with respect to a fixed reference frame, $\psi$ is the angle of the rotor relative to the board, and $\phi_{b}$ and $\phi_{f}$ are, respectively, the angles subtended by the back and front wheels (relative to the body). For simplicity, we restrict the front and back wheel motions so that $\phi_{b}=\phi=-\phi_{f}$. The rotor and wheels are actuated. The wheels provide nonholonomic constraints on the snakeboard's motions. However, because it is not fully constrained, the snakeboard can build up momentum and coast.

After feedback linearization on the directly controlled states, the equations of motion are:

$$
\begin{aligned}
\ddot{\psi} & =u_{1} \\
\ddot{\phi} & =u_{2} \\
\dot{g} & =g\left(-\mathcal{A}_{l o c}(r) \dot{r}+\left(\mathbb{I}^{c}(r)\right)^{-1} p\right) \\
\dot{p} & =2 J_{r} \cos ^{2}(\phi) \dot{\phi} \dot{\psi}-\tan (\phi) \dot{\phi} p
\end{aligned}
$$



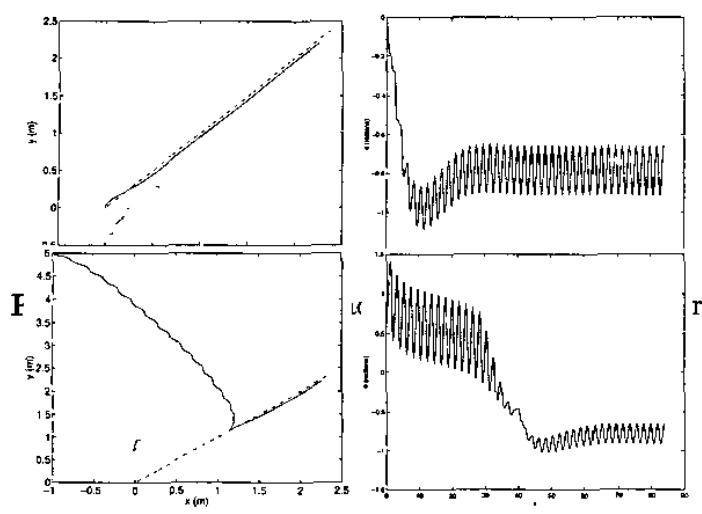

Figure 6: Trajectory Tracking for Discrete Crawler

where

$$
\begin{gathered}
\mathcal{A}_{\text {loc }}(r)=\left\{\begin{array}{c}
-\frac{J_{r}}{2 M l} \cos ^{2}(\phi) \tan (\phi) \\
0 \\
-\frac{J_{r}}{2 M l^{2}} \sin (2 \phi) \tan (\phi)
\end{array}\right\}, \\
\mathbb{I}^{c}(r)=\left\{\begin{array}{c}
\frac{1}{2 M l} \\
0 \\
-\frac{1}{2 M l} \tan (\phi)
\end{array}\right\}
\end{gathered}
$$

and $r=\left[\psi, \phi_{b}, \phi_{f}\right]^{T}$.

The control inputs consist of state feedback for the directly controlled states, coupled with periodic terms to influence the indirectly controlled states:

$u_{1}=-2 \psi-3 \dot{\psi}+\frac{1}{\epsilon} v^{1}\left(\frac{t}{\epsilon}\right), u_{2}=-2 \phi-3 \dot{\phi}+\frac{1}{2} \alpha_{2} \epsilon+\frac{1}{\epsilon} v^{2}\left(\frac{t}{\epsilon}\right)$

which results in the autonomous contribution,

$$
Y_{0}=-(2 \psi+3 \dot{\psi}) \frac{\partial}{\partial \dot{\psi}}-\left(2 \phi+3 \dot{\phi}-\frac{1}{2} \alpha_{2} \epsilon\right) \frac{\partial}{\partial \dot{\phi}} .
$$

The time-periodic control input contribution is,

$$
\begin{aligned}
& v^{1}(t)=-2 \operatorname{sign}\left(\alpha_{1}\right) \sqrt{\left|\alpha_{1}\right|} \cos (t) \\
& v^{2}(t)=-\frac{1}{2} \sqrt{\left|\alpha_{1}\right|} \cos (t)
\end{aligned}
$$

with $\alpha_{1}$ a free parameter. The averaged equations become,

$$
\begin{aligned}
\ddot{\psi} & =-2 \psi-3 \dot{\psi} \\
\ddot{\phi} & =-2 \phi-3 \dot{\phi}+\frac{1}{2} \alpha_{2} \epsilon \\
\dot{g} & =g\left(-\mathcal{A}_{l o c}(r) \dot{r}+\left(\mathbb{I}^{c}(r)\right)^{-1} p\right) \\
\dot{p} & =2 J_{r} \cos ^{2}(\phi) \dot{\phi} \dot{\psi}-\tan (\phi) \dot{\phi} p-2 J_{r} \cos ^{2}(\phi) \alpha_{1} .
\end{aligned}
$$

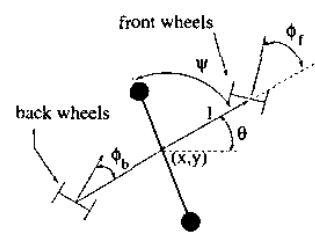

Figure 7: Model of the snakeboard.
The controllable subspace is then stabilized to the point $\left(0, \frac{1}{2} \alpha_{2} \epsilon\right)$, corresponding to a steering bias.

The group and velocity error in body coordinates are

$$
\begin{aligned}
& g_{\text {err }}=g_{f d b k}^{-1} g_{\text {des }} \\
& \xi_{\text {err }}=g_{d e s}^{-1} g_{\text {des }}^{\prime}+\operatorname{Ad}_{g_{f d b k}} g_{f d b k}^{\prime}
\end{aligned}
$$

where $g_{f d b k}$ is the feedback of the group variables, and $g_{d e s}$ is the desired trajectory. Noting that the term $\alpha_{1}$ corrects velocity and position along the relative equilibria, and the term $\alpha_{2}$ attracts trajectories to the relative equilibria curve, we define the parameters $\alpha_{1}$ and $\alpha_{2}$ according to Theorem 5 ;

$$
\begin{aligned}
& \alpha_{1}=-\frac{3}{16} x_{e r r}+\frac{3}{8} \xi_{e r r}^{1}, \\
& \alpha_{2}=\theta_{e r r}+\frac{3}{2} \dot{\theta}_{e r r}+\frac{1}{4} y_{e r r}+\frac{3}{4} \xi_{e r r}^{2} .
\end{aligned}
$$

At zero configuration error, the feedback terms $\left(\alpha_{1}, \alpha_{2}\right)$ and the oscillatory signals vanish. Hence, both the original and averaged systems share the same relative equilibria. Therefore, via Corollary 1 , the system exponentially stabilizes to the desired trajectory. Recall that state feedback corrections are determined at discrete times corresponding to the basic control oscillation. With $\epsilon=\frac{1}{3}$ this update occurs every $T=2 \pi / 3$ seconds.
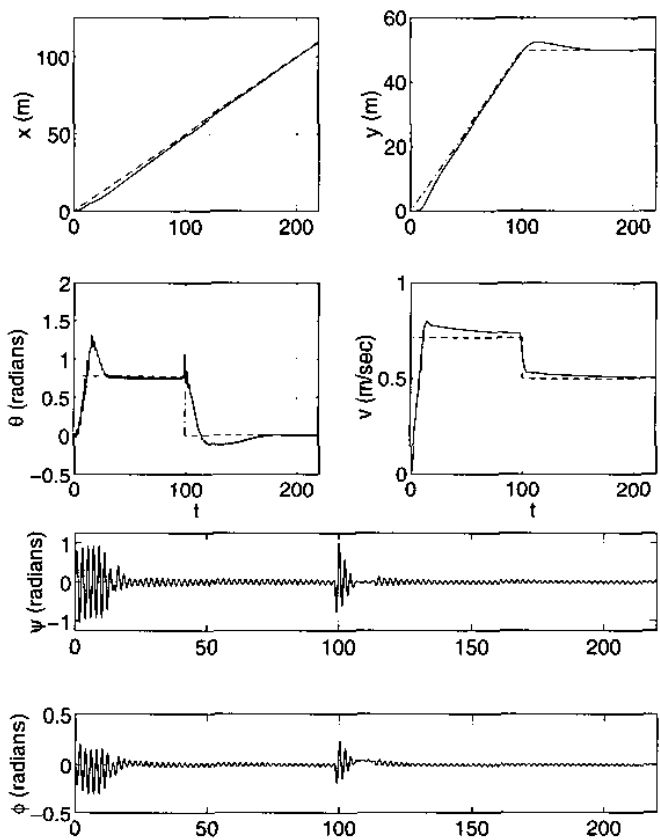

Figure 8: Trajectory Tracking results for the Snakeboard.

Stabilization is demonstrated in Fig. 8 where the system parameters are $M=8 \mathrm{~kg}, J_{r}=0.167 \mathrm{~kg} \mathrm{~m}^{2}, l=0.3 \mathrm{~m}$. The initial condition occurs at the origin, with zero initial velocity. The upper figures depict the evolution of the states $(x, y, \theta)$ and forward velocity, $v$, describing the relative equilibria in the body frame. The desired trajectory is the dash-dotted plot, and the actual trajectory is the solid plot. 




Figure 9: Trajectory tracking (snakeboard enlarged 6x)

The bottom two graphs show the rotor and steering angle histories. Fig. 9, shows snapshots (every 7 seconds) of the snakeboard's configuration during the first $50 \pi$ seconds. The grid lines are spaced 5 units apart. At time $t=100$, there is a discontinuous change in the desired trajectory's bearing and velocity. The oscillations decrease in amplitude as the error approaches zero. There is a small steady state error (which can be eliminated by the use of an integral feedback term).

\section{Conclusion}

Because of the periodic nature of biomimetic locomotory movement, averaging theory seems to be an excellent approach to rigorously understanding and simplifying the complex dynamics and control underlying biomimetic locomotion. We reviewed a recently developed generalized averaging theory, and the associated method for exponentially stabilizing underactuated mechanical systems via periodic inputs. Our results apply to an extremely large class of problems. For purposes of demonstration, we demonstrated the method on a simple biped model and the snakeboard. We have demonstrated this technique on models of amoeba-like robots [19], and experimentally on a robotic fish [15].

We are pursuing two extensions to this work. First, the averaged control equations appear to support the application of ideas from robust control theory. Second, we are trying to connect this work to the stratified configuration space approach for legged locomotion modelling $[4,5]$.

\section{References}

[1] Agračhev A. and Gamkrelidze R. The exponential representation of flows and the chronological calculus. USSR Sbornik, 35(6):727-785, 1978.

[2] Lewis A., Ostrowski J., Murray R., and Burdick J. Nonholonomic mechanics and locomotion: The snakeboard example. Proc. IEEE Int. Conf. Robotics and Automation, 1994.

[3] Bloch A.M., Krishnaprasad P.S., Marsden J.E., and Murray R.M. Nonholonomic mechanical systems with symmetry. CDS TEchnical Report 94-013, 1995.

[4] Goodwine B. and Burdick J.W. Controllability of kinematic control systems on stratified configuration spaces. IEEE Trans. Aut. Contr., 46(3):358-68, 2001.

[5] Goodwine B. and Burdick J.W. Motion planning for kinematic stratified systems with application to quasi-static legged locomotion and finger gaiting. IEEE Trans. Rob. Aut., 18(2):209-222, 2002.

[6] M Berkemeier. Modeling the dynamics of quadrupedal running. Int. J. Robotics Research, 16(9):971-985, 1998.

[7] M. Bühler and D. Koditschek. Analysis of a simplified hopping robot. Int. J. Robotics Research, 10(6):587-605, 1991.

[8] Bullo F. Averaging and vibrational control of mechanical systems. SIAM J. Contr. Opt., 41(2):542-62, 2002.

[9] Bullo F. and Lewis A. Kinematic controllability and motion planning for the snakeboard. Proc. IEEE Int. Conf. Rob. Aut., 2002.

[10] Bullo F. and Zefran M. On modelling and locomotion of hybrid mechanical systems with impacts. IEEE Conference on Decision and Control, 1998.

[11] Matsuno F., Ito K., and Takahashi R. Local accessibility and stabilization of an underactuated crawling robot with changing constraints. IEEE Int. Conf. Robotics and Automation, 2001.

[12] J. Furusho and A. Sano. Sensor-based control of a nine-link biped. Int. J. Robotics Research, 9(2):83-98, 1990.

[13] Ostrowski J. and Burdick J.W. The mechanics and control of undulatory locomotion. Int. J. Robot. Res., 17(7):683-701, 1998.

[14] Ito K., Matsuno F., and Takahashi R. Underactuate crawling robot. Int. Conf. on Intelligent Robots and Systems, 2001.

[15] Morgansen K.A., Vela P.A., and Burdick J.W. Trajectory stabilization for a planar carangiform robot fish. Proc. IEEE Int. Conf. Rob. Aut., 2002.

[16] Vela P.A. and Burdick J.W. Control of underactuated driftless systems using higher-order averaging. Sub. to 2003 Amer. Contr. Conf., 2002.

[17] Vela P.A. and Burdick J.W. A general averaging theory via series expansions. Sub. to 2003 Amer. Contr. Conf., 2002. [18] Vela P.A., Morgansen K.A., and Burdick J.W. Second order averaging methods for oscillatory control of underactuated mechanical systems. (submitted) SIAM J. Contr. Opt., 2002.

[19] Vela P.A., Morgansen K.A., and Burdick J.W. Underwater locomotion from oscillatory shape deformations. To appear, IEEE Conf. Dec. Contr., 2002.

[20] M.H. Raibert. Legged Robots that Balance. MIT Press, 1986.

[21] S.M. Song and K.J. Waldron. Machines that walk: the Adaptive Suspension Vehicle. MIT Press, 1989.

[22] Suzuki T. and Nakamura Y. Nonlinear control of a nonholonomic free joint manipulator with the averaging method. In IEEE Conf. Dec. Contr., pages 1694-1699, 1996.

[23] A.F. Vakakis, J.W. Burdick, and T.K. Caughey. An interesting strange attractor in the dynamics of a hopping robot. Int. J. Robotics Research, 10(6):606-618, 1991. 